\title{
Volcanic aerosol radiative properties
}

Andrew Lacis

\section{Large sporadic volcanic eruptions inject large amounts of sulfur bearing gases into the stratosphere which then get photochemically converted to sulfuric acid aerosol droplets that exert a radiative cooling effect on the global climate system lasting for several years.}

Volcanic aerosol consists mainly of concentrated sulfuric acid $\left(75 \% \mathrm{H}_{2} \mathrm{SO}_{4}\right)$, although the bulk of the erupted volcanic material consists of large ash particles which fall out rapidly, leaving the sulfur bearing gases to form the more enduring volcanic aerosol. It is this longer lasting aerosol that causes cooling of the global surface temperature (solar albedo effect), and warming of the lower stratosphere (greenhouse effect). More generally, the magnitude of the surface cooling, and the degree of stratospheric warming depend in detail on the aerosol composition, its optical depth, particle size, and the height of the aerosol in the stratosphere.

Aerosol radiative properties can be calculated accurately for any aerosol size and composition using Mie scattering theory, which is an exact theory of how light is scattered by homogeneous spherical particles. Figure $1 \mathrm{~A}$ shows that the effective cross-section (expressed per unit mass) of atmospheric aerosols can differ greatly relative to the particle's geometric size. According to Mie theory, particles exhibit their peak scattering efficiency at a characteristic size that depends on the aerosol composition. In moving toward ever larger sizes, the mass-specific cross-section diminishes inversely with size, while in the small particle limit (i.e. the Rayleigh limit where particles are much smaller than the wavelength of incident radiation), the effective cross-section for non-absorbing particles such as sulfuric acid, aluminum oxide, sea salt, sulfates, nitrates, goes to zero (thus making them invisible), while the strongly absorbing particles (e.g. black carbon), retain most of their peak absorption efficiency even as the particle size approaches zero.

\section{Effect of particle size}

For non-absorbing aerosols, the cross-section per unit mass is largest for aerosols with effective radii near $0.25 \mu \mathrm{m}$. Particle size is also important considering that aerosol residence time in the stratosphere is governed by particle fallout speed, which is proportional to the particle radius squared. In their formative stage, volcanic aerosols are exceedingly tiny, which translates into negligible fallout speed and a negligible scattering cross-section as dictated by the small particle Rayleigh limit of Mie theory. Mature volcanic aerosols tend to be in the $0.25 \mu \mathrm{m}$ effective radius size range, and at that size they have a stratospheric residence time of about a year at the nominal volcanic aerosol altitude of $25 \mathrm{~km}$. For strongly absorbing aerosols such as black carbon, the Rayleigh limit has minimal effect on the particle's absorption efficiency. For more exotic aerosol compositions, such as metallic aluminum (for which the imaginary refractive index is much larger than unity), the particle surface becomes reflective, resulting in scattering characteristics that are similar to non-absorbing aerosols (Fig. 1A). The peak radiative efficiency for both black carbon and metallic particles occurs at less than half of the particle radius compared to non-absorbing aerosols. Basically, absorbing aerosols can be effective in cooling the ground surface if their absorption occurs high in the stratosphere, but they produce strong local heating that adversely impacts stratospheric ozone.

The principal radiative effect of volcanic aerosols is the solar shortwave (SW) cooling of the surface temperature (green dashed line and left-hand scale, Fig. 1B) computed with zero feedback magnification for a single-layer aerosol at $27-29 \mathrm{~km}$ altitude (with a mass density of $0.01 \mathrm{gm}^{-2}, 75 \% \mathrm{H}_{2} \mathrm{SO}_{4} \mathrm{com}$ position) as the aerosol effective radius is varied from zero to $1 \mu \mathrm{m}$. The aerosol effect can also be expressed equivalently as $\mathrm{Wm}^{-2}$ of radiative forcing (blue and red dashed lines for the SW and net radiative forcings, right hand scale). Also shown at the bottom of Figure 1B by the dotted and solid green lines is the LW (greenhouse effect) forcing of the volcanic aerosol. Notably, the LW forcing
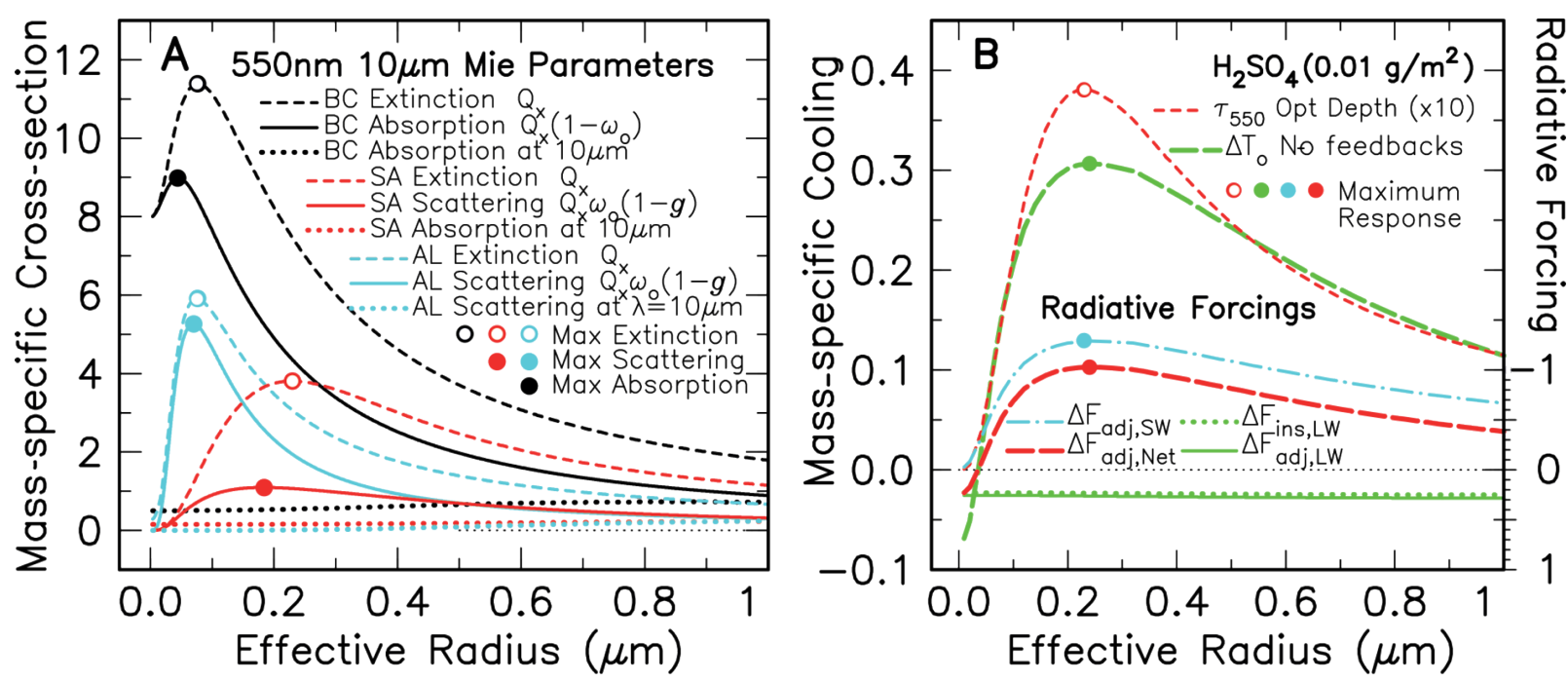

Figure 1: (A) Mass-specific cross-sections $\left(\mathrm{m}^{2} \mathrm{~g}^{-1}\right)$ at $550 \mathrm{~nm}$ and $10 \mu \mathrm{m}$ for black carbon (BC), sulfuric acid (SA), aluminum (AL) aerosols as functions of aerosol size. Dashed lines are Mie extinction efficiency factors $\mathrm{Q}_{x}$. BC absorption cross-section is solid black. Solid red and blue lines depict effective scattering cross-sections of SA and AL aerosols. Black and red dotted lines are BC and SA absorption cross-sections at $10 \mu \mathrm{m}$. Blue dotted line is AL scattering at $10 \mu \mathrm{m}$. (B) Mass-specific cooling for SA aerosol for mass density $0.01 \mathrm{gm}^{-2}$ as functions of aerosol size. Dashed red line is SA optical depth (x10). Long-dash green line depicts no-feedback surface temperature (cooling) change. Radiative forcings use right-hand scale. The long-dash red line depicts adjusted forcing with solar only (SW) component given by the dot-dash cyan line. The green lines are LW instantaneous and adjusted forcings. 


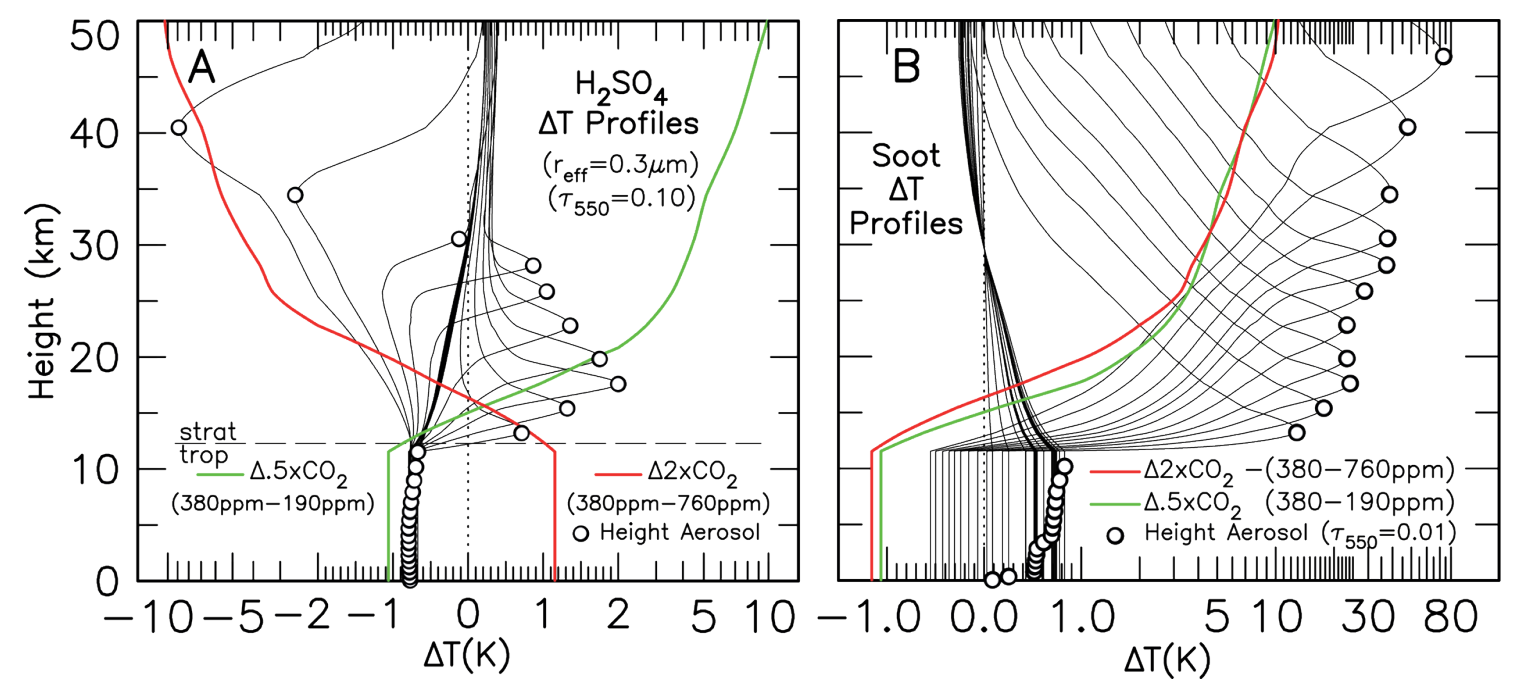

Figure 2: (A) Atmospheric heating and cooling profiles for sulfuric acid $\left(\mathrm{H}_{2} \mathrm{SO}_{4}\right)$ aerosol for optical depth $\mathrm{T}_{550}=0.10$ as functions of aerosol height. Aerosol height is denoted by the open circles. $\Delta T$ scale is linear from -2.0 to 2.0, logarithmic otherwise. Atmospheric temperature changes for $2 \times \mathrm{CO}_{2}$ ( $\left(r e d\right.$ line) and $0.5 \times \mathrm{CO}_{2}$ ( $(\mathrm{green}$ line), are included for comparison. (B) Atmospheric heating and cooling profiles for black carbon (soot) aerosol for optical depth $T_{550}=0.01$ as functions of height (open circles). The $\triangle T$ scale is linear from -1.0 to 1.0, otherwise logarithmic. For comparison, the temperature response for $2 \mathrm{xCO}_{2}$ and $0.5 \times \mathrm{CO}_{2}$ are plotted as a negative temperature change.

is virtually independent of particle size. This happens because sulfuric acid aerosol is strongly absorbing at thermal wavelengths, and for the size range shown, the aerosol is effectively within the Rayleigh limit relative to the wavelength of thermal radiation (hence there is only minimum change in the LW absorption cross-section with size).

A simple estimate of the aerosol potential climate impact is the instantaneous radiative forcing $\Delta \mathrm{F}_{\text {ins }}$ which is just the change in $\mathrm{LW}$ net flux at the tropopause calculated with and without the aerosol. A more accurate estimate is the adjusted radiative forcing $\Delta \mathrm{F}_{\text {adj' }}$ which is obtained by allowing the stratosphere to equilibrate with ground and tropospheric temperatures kept constant. The radiative/convective equilibrium response can also be expressed as a surface temperature $\left(\Delta \mathrm{T}_{0}\right)$ change representing the radiative forcing in temperature equivalent units without feedback effects being included. To estimate the full-equilibrium surface temperature response $\left(\Delta \mathrm{T}_{\mathrm{eq}}\right)$, with climate system feedback contributions included, $\Delta T$ 。 is multiplied by a climate feedback factor, which, in the case of the GISS climate GCM with $2.7^{\circ} \mathrm{C}$ sensitivity for $2 \mathrm{xCO}_{2}$, is $f \sim 2.3$ (Lacis and Mishchenko 1995).

The perception that if aerosols are very small, they will have little or no LW radiative effect is not accurate. This is because for strong LW absorption, e.g. $\mathrm{H}_{2} \mathrm{SO}_{4^{\prime}}$ the absorption cross-section remains largely independent of particle size in the Mie scattering Rayleigh limit for absorbing aerosols. Thus, volcanic $\mathrm{H}_{2} \mathrm{SO}_{4}$ aerosols will produce some surface warming when the aerosol size is smaller than $r_{\text {eff }}=0.04 \mu \mathrm{m}$. Also, when $\mathrm{H}_{2} \mathrm{SO}_{4}$ aerosols are larger than $2.2 \mu \mathrm{m}$, the LW radiative heating contribution exceeds the SW scattering component to produce surface warming.

\section{Effect of vertical aerosol distribution}

Typically, volcanic aerosols absorb little SW solar radiation. Hence, the vertical distribution of the aerosol has minimum impact on the surface cooling. However, the aerosol height does have an important bearing on the stratospheric heating. Figure $2 \mathrm{~A}$ demonstrates the radiative effect of volcanic aerosol height on atmospheric temperature by stepping the $\mathrm{H}_{2} \mathrm{SO}_{4}$ aerosol layer-by-layer from the ground to the mesosphere. The largest heating occurs in the lower stratosphere, where the stratosphere is the coldest, and where the absorbed LW radiation exceeds the local LW emission by the largest margin. $\mathrm{H}_{2} \mathrm{SO}_{4}$ does absorb strongly at near-IR wavelengths beyond $3 \mu \mathrm{m}$, but there is little solar energy there to be absorbed in this spectral region (Lacis et al. 1992).

At altitudes higher than about $30 \mathrm{~km}$, where stratospheric temperatures are comparable to ground surface temperatures, the $\mathrm{H}_{2} \mathrm{SO}_{4}$ aerosols exhibit substantial stratospheric cooling. However, the radiative forcing that drives the global surface cooling $\left(\Delta T_{0}\right)$ is largely independent of height, and is equal to approximately $-0.7^{\circ} \mathrm{C}$ (Fig. 2A). For comparison, the equilibrium response of stratospheric temperature to $0.5 \times \mathrm{CO}_{2}(380-190$ $\mathrm{ppm}$ ) and $2 \mathrm{xCO}_{2}(380-760 \mathrm{ppm})$ is shown by the heavy green and red lines, respectively.

In contrast to the behavior of $\mathrm{H}_{2} \mathrm{SO}_{4}$ aerosols, Figure $2 \mathrm{~B}$ shows the atmospheric heating and cooling profiles characteristic for strongly absorbing black carbon (soot) aerosol. Because of its greater surface cooling efficiency, optical depths $\left(T_{550}=0.01\right)$ an order of magnitude smaller are used for soot than for $\mathrm{H}_{2} \mathrm{SO}_{4}$. A principal characteristic of strongly absorbing aerosols is their strong tropospheric and surface warming if the soot aerosol is deployed within the troposphere. Significant cooling of the ground surface is possible when the soot aerosol is deployed at high altitude, approaching $-1.86 \mathrm{Wm}^{-2}$ surface cooling for soot deployed at 44-50 $\mathrm{km}$ altitude. This behavior is similar to what happens in nuclear winter scenarios when the thermal back-warming from the stratosphere is much diminished by the increasing height of the aerosol. Predictably, soot that is deployed high in the stratosphere will generate extreme stratospheric heating, which is an undesirable bi-product. In summary, the radiative impact of soot on the surface temperature is strong heating for soot deployed within the troposphere, but when deployed at high altitudes in the stratosphere, soot can also produce strong cooling of the ground surface.

\section{Considerations for geoengineering applications}

Large volcanic eruptions have been valuable radiative forcing experiments to test climate model response to stratospheric aerosol injections. Based on this, aerosols have also been suggested for geoengineering applications to counteract global warming. For this purpose, the non-absorbing aerosols would appear to be the logical choice in that they could mimic the radiative effects of naturally occurring volcanic aerosols. But among the drawbacks would be the need to maintain millions of tons of scattering aerosols in the stratosphere since particles that are large enough to scatter efficiently tend to fall out from the stratosphere within a year. Thus, for non-absorbing aerosols, maintaining the optimum aerosol size would be critical - if the aerosols are too large, they fall out too fast, if they are too small, their scattering efficiency becomes too small. The results presented here also demonstrate that strongly absorbing aerosols could efficiently cool the ground surface, but that the accompanying stratospheric heating would be exceedingly large.

\section{AFFILIATIONS}

NASA Goddard Institute for Space Studies, New York, USA

\section{CONTACT}

Andrew Lacis: andrew.a.lacis@nasa.gov

\section{REFERENCES}

Lacis AA, Mishchenko MI (1995) In: Charlson RJ, Heintzenberg J (Eds) Aerosol Forcing of Climate. Wiley, 11-42

Lacis A et al. (1992) Geophys Res Lett 19: 1607-1610 